\title{
Ion Beam Emittance for an Ion Source with a Conical Hot Cavity
}

\author{
M. TUREK \\ Institute of Physics, Maria Curie Sklodowska University in Lublin \\ Pl. M. Curie-Sklodowskiej 1, 20-031 Lublin, Poland
}

\begin{abstract}
The emittance of stable and short-lived nuclide ion beams produced in the conically shaped hot cavity ion sources is calculated. Changes of beam emittance with the half-life period as well as the average sticking time are under investigations. Calculations are done for different lengths of the hot cavity in order to demonstrate both advantages and drawbacks of each shape. Influence of the extraction channel geometry (its diameter and length) on the ion beam quality is studied. A scaled efficiency, ion beam brightness analogon, is used to compare different ion source configurations.
\end{abstract}

DOI: 10.12693/APhysPolA.136.329

PACS/topics: 07.77.Ka, 07.05.Tp, 34.35.+a, 41.75.Ak, 41.75.Cn

\section{Introduction}

Intense high-quality particle beams are corner-stones in many fields of experimental physics (including highenergy and accelerator physics, nuclear spectroscopy, ion beam modification of materials etc.). It should be kept in mind that not only the intensity of the ion beam is a crucial parameter but also its quality measured e.g. by beam divergence (or convergence) and its spread. Computer simulations are a commonly used tool widely applied to design efficient ion sources, ion guns, injectors, or transmission lines characterized by low transverse emittance [1-6].

Hot cavity ion sources have been used in different ISOL (Isotope Separation On-Line) facilities $[7,8]$ for several decades $[9,10]$, mainly due to their numerous advantages as high ionisation efficiency, excellent beam purity, and low energy spread of the produced beam. It should be also stressed that the short time atoms stay in the ioniser is crucial in the case of radioactive nuclides characterised by short half-lives. The crucial part is that an ioniser is usually made of refractory metal and heated to a very high working temperature ( $\sim 2500 \mathrm{~K}$ or more) either by electron bombardment or ohmically [11, 12]. The most popular form is a semi-opened tube but the spherical cavities are also applied in some designs [13,14]. It should be mentioned that in the ISOL facilities the ioniser is either connected to a target by a kind of transfer line $[13,15]$ or the specially designed ioniser could be simultaneously the irradiated target where new isotopes are produced and then released into the ioniser cavity [16].

Several analytical models of processes taking place in hot cavity ion sources have been proposed for years [17-19] (for more details see [20]). Also the diffusion of nuclides in target and effusion in surface ion sources

e-mail: mturek@kft.umcs.lublin.pl was addressed in [21, 22]. Over last years a numerical model of ionization in hot cavity ion source has been proposed $[20,23,24]$ taking into account not only the fundamental ionisation mechanism (surface ionization) but also influence of electron impact ionization $[25,26]$. In its early form the model was suitable for stable and longlived isotopes. Radioactive decay and delays, due to the nuclide diffusion towards the target surface and sticking to hot walls and effusion, were implemented $[20,27]$ which made the model applicable also for short-lived isotopes. The most popular tubular cavities were considered as first, but then the model was extended also to spherical and hemispherical cavities [20, 28, 29]. Recently [30,31] the hot cavity having a shape of a truncated cone has been proposed. Such a shape could be considered as transition between the elongated shapes of tubular ionisers and more compact spherical shapes having advantage for short-lived isotopes. On the other hand, the conical cavity is by far easier to produce than a spherical one, as the refractory metals such as $\mathrm{W}, \mathrm{Mo}$, or Ta are very hard to machine. In the paper [30] the advantage of elongated cavity shapes over the compact ones was proved in the case of stable isotopes. The twofold increase of ionisation efficiency is especially important for the substances hard to ionise. On the other hand, compact shape prevails for very short-lived isotopes as the average time a particle stays in the compact cavity is shorter than that for the elongated one.

The brief description of the numerical model used for simulation as well as the description of rms emittance formulae is given in the paper for the sake of completeness. The results of emittance calculations for different elongations of the cavity in the case of stable isotopes are presented and discussed. Dependences of beam emittance on nuclide surface ionization probability, half-life period, and its average sticking time obtained for different cavity configurations are also considered. Influence of the extraction opening geometry i.e. the extraction opening radius and the length of the extraction channel 
is studied. A previously introduced [32] concept of scaled efficiency is used in order to compare beam quality for different extraction opening configurations as well as isotope half-lives.

\section{Numerical model}

The numerical code is designed to follow the trajectories of particles inside the hot ioniser cavity. In the considered case the cavity is conically-shaped. It is schematically shown in Fig. 1 . The cavity has the height $h$, as well as upper and lower base radii $r$ and $R$, respectively. The flat (for the sake of simplicity) extraction electrode is placed at the distance $d$ from the extraction aperture. The simulation region is covered by 800x100x100 rectangular numerical grid. The cell sizes are chosen as $\Delta x=\Delta y=\Delta z=0.05 \mathrm{~mm}$. The successive over-relaxation method is used to solve the discrete Laplace equation and to find the electrostatic potential, as in [19, 20]. One should bear in mind that the shapes and potentials of electrodes impose boundary conditions of the Laplace equation. The $4^{\text {th }}$ order Runge-Kutta method is applied to integrate the classical equation of motion and determine particle trajectories. The electric field acting on a particle is calculated by a linear interpolation of $E$ values at nearest grid nodes. Particles are started from the internal surface of the cavity assuming random distribution of their directions. The ioniser temperature $T$ determines particle velocity. As the typical pressures inside the cavity are as low as $10^{-4}$ mbar [33] (the typical mean path between collisions is longer than $1 \mathrm{~m}$ ), it is justified to neglect the partcile-particle collisions.



Fig. 1. A schematic view of the considered geometry of the ion source.

A particle could be ionised or neutralized during the collision with a hot ioniser wall with a probability described by the ionisation coefficient $\beta$. It is very closely related to the commonly used ionisation degree

$$
\beta=\alpha /(1+\alpha) \text {. }
$$

On the other hand, the $\alpha$ coefficient is usually defined as the ratio of the numbers of ions and neutral atoms leaving the hot surface. This magnitude could be calculated using the Saha-Langmuir formula

$$
\alpha=G \exp \left(-\left(V_{i}-\varphi_{e}\right) / k_{\mathrm{B}} T\right),
$$

where $V_{i}$ and $\varphi_{e}$ are the ionisation potential of an atom and the work function of the ioniser, respectively. The $G$ coefficient depends on the ioniser surface and the ionised atom properties, including the reflection coefficient and statistical weights of the ionised and neutral states. It is assumed that a particle sticks to the surface for a time period, which is determined using the typical Monte Carlo approach for every collision

$$
t_{\text {stick }}=-\tau_{s} \ln \mathrm{RND},
$$

where $\tau_{s}$ is the average sticking time and RND is the pseudorandom number in the range $(0,1)$. Typical values of $\tau_{s}$ could be found e.g. in [34]. The model takes into account radioactive decay of the primary nuclide. Each nucleus of that kind undergoes decay after the time $t$ dec, which is calculated according to the formula

$$
t_{\mathrm{dec}}=-\tau_{1 / 2} \ln R N D,
$$

where $\tau_{1 / 2}$ is the half-life period of the considered isotope. The code follows the particles trajectories until they pass the extraction opening and counts the numbers of primary and secondary nuclides ions $\left(N_{p+}\right.$ and $\left.N_{s+}\right)$ as well as the number of neutrals $\left(N_{p 0}\right.$ and $\left.N_{s 0}\right)$. The ion source ionization efficiency is defined as the ratio

$$
\beta_{s}=\frac{N_{p+}}{N_{p+}+N_{s+}+N_{p 0}+N_{s 0}} .
$$

This efficiency differs much from the ionisation probability $\beta$ defined for a single particle wall collision and these magnitudes should not be mixed up. Multiple collisions of particles with the hot ioniser surface may result in the increase of the total ion source efficiency defined by Eq. (5) to the values much higher than those predicted by the Saha-Langmuir law.

For the sake of completeness a short resume of the beam emittance is given below. A beam formed by $N$ particles could be represented by a single point in the $6 N$-dimensional canonical phase space composed of each particle's $\left(x, y, z, p_{x}, p_{y}, p_{z}\right)$ coordinates. Alternatively, one can use $N$ points in 6 -dimensional phase space instead. As long as all particles move collectivelly in approximately the same direction (say, along the $z$ axis), it is reasonable to treat longitudinal and transversal motions separately. The momentum along the beam axis $\left(p_{z}\right)$ is much greater than the transversal component. Therefore, instead of the momenta, it is convenient to consider

$$
x^{\prime}=\frac{p_{x}}{p_{z}} \text { and } y^{\prime}=\frac{p_{y}}{p_{z}}
$$

The fractional emittances (transversal) are defined as the surface of the ellipses containing rather an arbitrarily chosen part of the beam (usually 99\%, 95\%, or 90\%) in the $x x^{\prime}$ and $y y^{\prime}$ subspaces (often divided by $\pi$ ).

The code calculates the r.m.s. emittance as it is more convenient in the numerical simulations. It is defined $[35,36]$ as: 


$$
\begin{aligned}
& \varepsilon_{x, \mathrm{rms}}=\sqrt{\left\langle x^{2}\right\rangle\left\langle x^{\prime 2}\right\rangle-\left\langle x x^{\prime}\right\rangle^{2}} \\
& \text { and } \varepsilon_{y, \mathrm{rms}}=\sqrt{\left\langle y^{2}\right\rangle\left\langle y^{\prime 2}\right\rangle-\left\langle y y^{\prime}\right\rangle^{2}}
\end{aligned}
$$

where brackets indicate the average over the whole ensemble. It should be kept in mind that several authors suggest multiplying rms emittance by factor 4 in order to take into account also the particles outside the rms ellipse.

\section{Results}

Calculations of ion beam emittance were made in the case of stable isotopes $\left(\tau_{1 / 2}=0\right)$ at first. The ioniser geometry is described by $R=2.4 \mathrm{~mm}$, $r=0.2 \mathrm{~mm}, u=0.1 \mathrm{~mm}$, and $r_{\text {ext }}=0.5 \mathrm{~mm}$. The extraction electrode potential was $V_{e x t}=2 \mathrm{kV}$ and its distance from the extraction hole was chosen as $d=0.2 \mathrm{~mm}$. Simulations were done using 200000 (unless otherwise specified) of the test particles of 150 a.m.u. mass. The simulation timestep was $10^{-8} \mathrm{~s}$. The ioniser temperature was set to $k_{\mathrm{B}} T=0.3 \mathrm{eV}$. The results for cavities of different lengths $h$ are shown in Fig. 2. As one can see the beam emittance decreases with the $\beta$ parameter independently of the cavity length. This is related to the fact that particles of the substances that are easier to ionise undergo a smaller number of collisions with the cavity walls. Moreover, the longer is the cavity, the higher is the beam quality. In the case of short cavities the particles reach the extraction region with a larger transversal momentum component as it was observed in the case of hemispherical cavities [32]. This results in larger emittance values exceeding $50 \mathrm{~mm}$ mrad in the case of $h=30 \mathrm{~mm}$. On the other hand, one can observe that erms tends to the limit of $37 \mathrm{~mm}$ mrad with the increasing $h$ for small values of $\beta$ parameter $(\beta<0.1)$.

Changes of ion beam emittance with the nuclide halflife were also studied. The values of $\tau_{1 / 2}$ changed in the range from $10 \mathrm{~ms}$ to $10 \mu \mathrm{s}$. Two opposite cavity

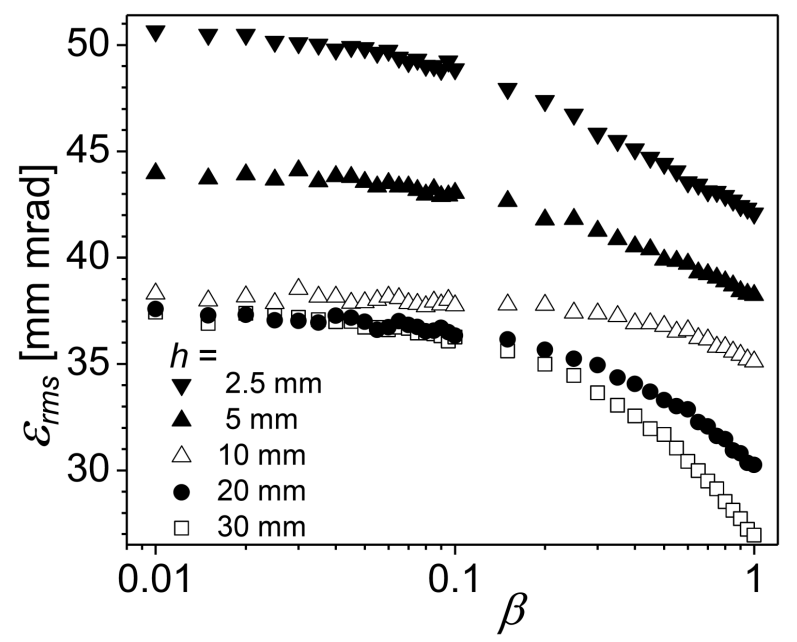

Fig. 2. The $\varepsilon_{\mathrm{rms}}(\beta)$ curves for different values of cavity length $h$ in the case of stable isotopes. configurations were under consideration: the elongated one (E) i.e. the case of $h=30 \mathrm{~cm}$ and the compact one (C) characterised by $h=2.5 \mathrm{~mm}$. The calculation results are shown in Fig. 3. For the longest $\tau_{1 / 2}$ the beam emittance is $\sim 38 \mathrm{~mm}$ mrad in the case (E) while it exceeds $50 \mathrm{~mm} \mathrm{mrad}$ for the compact cavity. One can see that $\varepsilon_{\mathrm{rms}}(\beta)$ curves behave in two different ways in each of the two cases. In the case $(\mathrm{C})$ one observes lowering of $\varepsilon_{\mathrm{rms}}(\beta)$ with the decreasing $\tau_{1 / 2}$ (improving the beam quality) while the reverse trend could be seen for the configuration $(\mathrm{E})$. It should be noticed that the $\varepsilon_{\mathrm{rms}}(\beta)$ curves are much flatter (particularly for $\beta<0.1$ ) compared to the case of (hemi)spherical cavities [32].



Fig. 3. The $\varepsilon_{\mathrm{rms}}(\beta)$ curves for different nuclide half-life periods $\tau_{1 / 2}$ (sticking neglected) for the two different shapes of the cavity.

Influence of average sticking time $\tau_{s}$ on the beam emittance was also investigated (Fig. 4). Calculations were done for $\tau_{1 / 2}=0.1 \mathrm{~s}$ and $\tau_{s}$ changing in the range from $10 \mu \mathrm{s}$ up to $100 \mathrm{~ms}$. All other simulation parameters were kept the same as in the previously considered case. Again the (E) configuration prevails with the emittance below $40 \mathrm{~mm} \mathrm{mrad}$. The influence of $\tau_{s}$ on the shift of $\varepsilon_{\mathrm{rms}}(\beta)$ curves is quite different for the two considered 
configurations. In the case of compact cavity (C) emittance decreases to $40 \mathrm{~mm}$ mrad and even below as the average sticking time rises. The $\varepsilon_{\mathrm{rms}}(\beta)$ curves are bunched together except for the curve for the shortest $\tau_{s}$, the emittance values for different sticking times do not differ more than by $5 \mathrm{~mm} \mathrm{mrad} \mathrm{(for} \beta<0.1$ ). However, it could be noticed that the ordering of the curves (for $\beta>0.1$ ) is inverse, compared to the case (E).

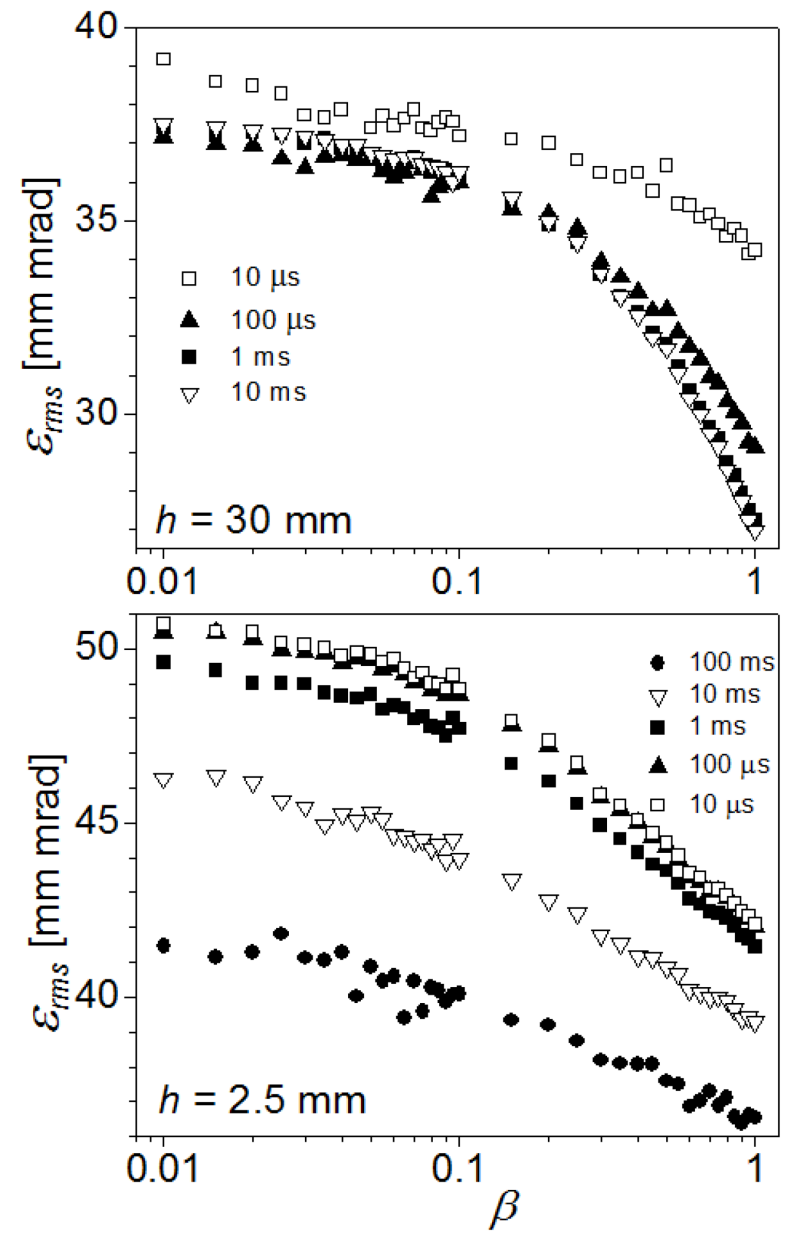

Fig. 4. The $\varepsilon_{\mathrm{rms}}(\beta)$ curves for different average sticking times $\tau_{s}$ and the two different shapes of the cavity.

The changes of the beam emittance obtained for $\beta=0.1$ with the extraction hole radius $r_{\text {ext }}$ are shown in Fig. 5. Calculations were performed for the two cavity geometries and two sets of timescales (1) $\tau_{1 / 2}=0.1 \mathrm{~s}$ and $\tau_{s}=1 \mathrm{~ms}(2) \tau_{1 / 2}=0.1 \mathrm{~ms}$ and $\tau_{s}=0.001 \mathrm{~ms}$. It should be mentioned that the simulations for the shorter timescales were done using 400000 particles for better statistics. One can see different behaviours of $\varepsilon_{\mathrm{rms}}\left(r_{\text {ext }}\right)$ curves for the two considered cavity shapes. In the case of elongated cavity the emittance rises with $r_{\text {ext }}$ up to $\sim 37 \mathrm{~mm} \mathrm{mrad} \mathrm{for} r_{\text {ext }}$ near $0.5 \mathrm{~mm}$ and then saturation of that trend is observed. On the other hand, $\varepsilon_{\text {rms }}$ grows constantly with $r_{\text {ext }}$ reaching the values above $90 \mathrm{~mm} \mathrm{mrad}$ for $r_{\text {ext }}=1 \mathrm{~mm}$ and no saturation is expected. This is due to the earlier mentioned fact that in the case of the flat conical cavity a lot of ions reach the extraction region with a relatively huge transversal component of momentum, as they are produced in the peripheral parts of the cavity. It should be noticed that the $\varepsilon_{\mathrm{rms}}\left(r_{\mathrm{ext}}\right)$ curves are very similar for the two sets of timescales.

In analogy to the beam brightness, another frequently applied figure of merits for the ion source, a scaled efficiency, was introduced in the previous paper [32]:

$$
B=\frac{\beta_{s}}{\varepsilon_{x, \mathrm{rms}} \varepsilon_{y, \mathrm{rms}}},
$$

This magnitude introduces a relation of the efficiency of the ion source to the phase space volume of the beam it produces. Figure 5 shows also the changes of B with $r_{\text {ext }}$. For longer timescales the $B\left(r_{\text {ext }}\right)$ curves behave in a quite similar way decreasing with the extraction radius, however, the curve for the (E) configuration is almost flat. The (E) configuration prevails in the whole considered $r_{\text {ext }}$ range, the scaled efficiency is 5-1-0 times larger than that for (C) shape. This changes for the isotopes with a shorter half-life period. One can see that the compact cavity (short effusion time) with small extraction opening (low emittance) results in the highest performance. For larger $r_{\text {ext }}$ values the saturation of $B\left(r_{\text {ext }}\right)$ curve for the (E) shape could be observed, as both efficiency [31] and the beam emittance have a constant value.

It can be expected that the second parameter describing the extraction opening geometry, namely the length of extraction channel $u$ influences the ion source performance substantially. On one hand, the emittance of the produced ion beam should decrease with $u$ because the longer is the extraction channel, the more effectively one gets rid of particles carrying a large transversal momentum. On the other hand, the ion source degrades with $u$ because of the fact that the long extraction channel leads to worse penetration of the extraction field. Figure 5 presents the results of the beam emittance and scaled efficiency calculations for $u$ changing up to $1 \mathrm{~mm}$ performed for the two cavity geometries and the same timescale sets as previously. As it was expected, the beam emittance decreases very fast with the extraction channel length. The $\varepsilon_{\mathrm{rms}}(u)$ curves for the $(\mathrm{C})$ configuration are much steeper that those obtained for the elongated one. The behaviour of emittance is more or less the same for the two timescale sets. The order of $B(u)$ curves is, however, completely different for shorter and longer $\tau_{1 / 2}$. The scaled efficiency grows with the length of the extraction channel. In the case of $\tau_{1 / 2}=0.1 \mathrm{~s}$ the elongated configuration prevails. In the case of shorter $\tau_{1 / 2}$ the compact cavity is much more efficient [31] and the longer extraction channel allows removal of particles with a large transversal momentum from the beam. The scaled efficiency for the $(\mathrm{C})$ shape is $\sim 4$ times larger than that obtained using the elongated cavity. In the (E) case rather saturation of $B(u)$ could be observed. 


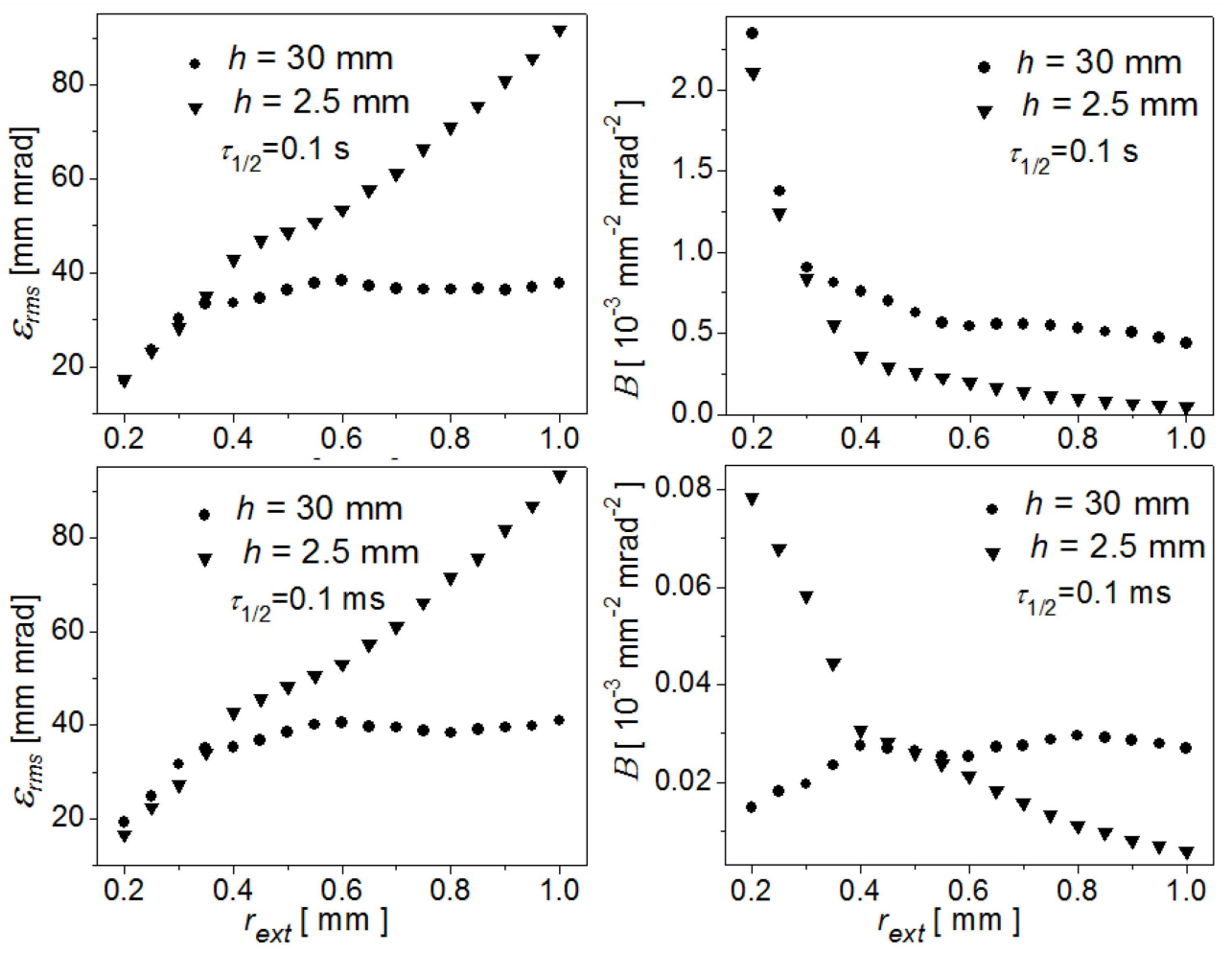

Fig. 5. The evolution of beam emittance and scaled efficiency with the extraction opening radius calculated for different cavity configurations and timescale sets.
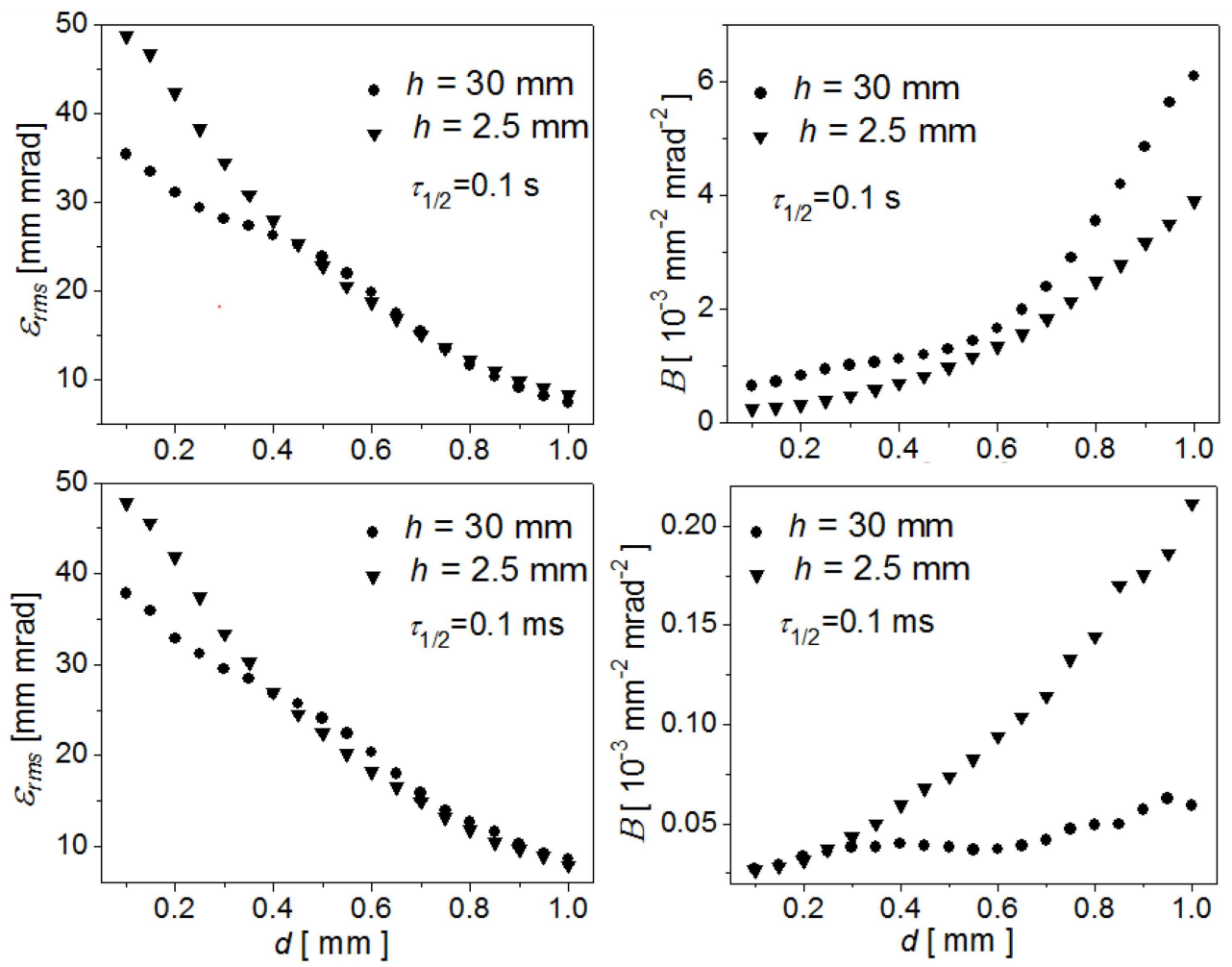

Fig. 6. The evolution of beam emittance and scaled efficiency with the extraction length calculated for the same configurations and timescale sets as in Fig 5. 


\section{Conclusions}

Emittance calculations for the particle beams produced in the conical hot cavity ion source were presented in the paper. The beam emittance calculated for the stable isotopes decreases with the cavity elongation. Changes of ion beam emittance with the nuclide half-life were also investigated for two different cavity configurations. It was found that in the case of the compact cavity beam emittance increases with the half-life period while the inverse ordering could be seen for the elongated configuration. Also the evolution of emittance with the average sticking time is different for the two considered cavity shapes. The elongated cavity produces an ion beam of better quality but the beam emittance changes much with $\tau_{s}$. On the other hand, in the case of compact cavity the emittance does not depend much on $\tau_{s}$. Influence of the geometry channel on the produced beam quality was studied. The beam emittance grows with the radius of the extraction opening for the compact cavity while the saturation of $\varepsilon_{\mathrm{rms}}\left(r_{\text {ext }}\right)$ curves is observed for $r_{\text {ext }}>0.5$ in the case of elongated ioniser cavity. The advantages of compact cavity with small extraction opening in the case of short-lived isotopes are demonstrated using the concept of scaled efficiency. The changes of scaled efficiency with the length of the extraction channel were also tested. While the elongated cavity works better in the case of stable and long-lived isotopes, the compact cavity with a long extraction channel could be several times more efficient in the case of short-lived isotopes.

\section{References}

[1] I. Dornmair, K. Floettmann, A.R. Maier, Phys. Rev. ST Accel. Beams 18, 041302 (2015).

[2] M.M. Abdelrahman, S.G. Zakhary, Braz. J. Phys. 39, 275 (2009).

[3] D. Winklehner, D. Todd, J. Benitez, M. Strohmeier, D. Grote, D. Leitner, J. Instrum. 5, P12001 (2010).

[4] H.J. Kwon, H.S. Kim, D.I. Kim, J.H. Jang, Y.S. Cho, J. Kor. Phys. Soc. 56, 1998 (2010).

[5] J.V. Mathew, S.V. Rao, R. Pande, P. Singh, Rev. Sci. Instrum. 86, 073306 (2015).

[6] P. Chen, R. Yi, D. Yu, in: Proc. IEEE Particle Accelerator Conference, Albuquerque (NM) 2007, p. 3684.

[7] V.N. Panteleev, Rev. Sci. Instrum. 75, 1602 (2004).

[8] U. Köster, O. Arndt, E. Bouquerel, V.N. Fedoseyev, H. Franberg, A. Joinet, C. Jost, I.S.K. Kerkines, R. Kirchner, The TARGISOL Collaboration, Nucl. Instrum Meth B 266, 4229 (2008).

[9] G.J. Beyer, E. Herrmann, A. Piotrowski, V.I. Raiko, H. Tyroff, Nucl. Instrum. Meth. 96, 437 (1971).
[10] P.G. Johnson, A. Bolson, C.M. Henderson, Nucl. Instrum. Meth. 83, 106 (1973).

[11] L. Zhai, H. Deng, G. Wei, Z. Li, C. Wang, X. Li, G. Zhou, Y. Su, Z. Zhang Z, International Journal of Mass Spectrometry 305, 45 (2011).

[12] C. Eléon, P. Jardin, G. Gaubert, M. Saintlaurent, J. Alcantaranunez, R. Alvesconde, Nucl Instrum Meth B 266, 4362 (2008).

[13] G.D. Alton, Y. Liu, H. Zaim, S.N. Murray, Nucl. Instrum. Meth. B 211, 425 (2003).

[14] P.A. Hausladen, D.C. Weisser, N.R. Lobanov, L.K. Fifield, H.J. Wallace, Nucl. Instrum Meth. B 190, 402 (2002).

[15] G.D. Alton, Y. Zhang, Nucl. Instrum. and Meth A 539, 540 (2005).

[16] V.G. Kalinnikov, K.Y. Gromov, M. Janicki, Y.V. Yushkevich, A.W. Potempa, V.G. Egorov, V.A. Bystrov, N.Y. Kotovsky, S.V. Evtisov, Nucl. Instr. and Meth. B 70, 62 (1992).

[17] A. Latuszyński, V.I. Raiko Nucl. Instrum. Meth. 125, 61 (1975).

[18] V.P. Afanas'ev, V.A. Obukhov, V.I. Raiko, Nucl. Instrum. Meth. 145, 533 (1977).

[19] A. Latuszyński, K. Pyszniak, A. Droździel, M. Turek, D. Mączka J. Meldizon, Vacuum 81, 1150 (2007).

[20] M. Turek, Vacuum 104, 1 (2014).

[21] Y. Zhang, G.D. Alton, J. Vac. Sci. Techn. A 23, 1558 (2005)

[22] B. Mustapha, J.A. Nolen, Nucl. Instrum. Meth. A 521, 59 (2004).

[23] M. Turek, K. Pyszniak, A. Drozdziel, J. Sielanko, Vacuum 82, 1103 (2008).

[24] M. Turek, A. Drozdziel, K. Pyszniak, D. Maczka, B. Slowinski, Rev. Sci. Instrum. 83 (2012) 023303.

[25] M. Turek, K. Pyszniak, A. Droździel, Vacuum 83, S260 (2009).

[26] M. Turek, Acta Phys. Pol. A 125, 1384 (2014).

[27] M. Turek, Acta Phys. Pol. A 123, 847 (2013).

[28] M. Turek, Acta Phys. Pol. A 120, 188 (2011).

[29] M. Turek, Acta Phys. Pol. A 128, 935 (2015).

[30] M. Turek, Acta Phys. Pol. A 132, 259 (2017).

[31] M. Turek, Przeglad Elektrotechniczny 94, 66 (2018).

[32] M. Turek, Acta Phys. Pol. A 128, 931 (2015).

[33] P.V. Duppen, Lect. Notes Phys. 700, 37 (2006).

[34] B. Eichler, S. Htibener, H. Rossbach, Zentralinstitut fuer Kernforschung Rossendorf Report Reports 560 and 561, 1985.

[35] F.J. Sacherer, IEEE Trans. Nucl. Sci. 18, 1105 (1971).

[36] R. Keller, J.D. Sherman, P. Allison, IEEE Trans. Nucl. Sci. 32, 2579 (1985). 